\section{References}

1. Noyez L, Lacquet LK. Recycling of the internal mammary artery in coronary reoperation. Ann Thorac Surg. 1993;55:597-9.

2. Antona C, Parolari A, Zanobini M, Arena V, Biglioli P. Midterm angiographic study of five recycled mammary arteries during four coronary redos. Ann Thorac Surg. 1966;61:702-5.

3. Velebit V, Maurice JP. Recycling of mammary arteries [Letter]. Ann Thorac Surg. 1996;62:947-9.
4. Noirhomme PH, Underwood MJ, El Khoury GA, et al. Recycling of arterial grafts during reoperative coronary artery operations. Ann Thorac Surg. 1999;67:641-4.

5. Scioti G, Cabib M, Balbarini A, et al. Late patency of recycled internal mammary artery: verification by Doppler echocardiography and coronary angiography. Tex Heart Inst J. 1999;26:303-5.

6. Uwabe K, Endo M, Kurihara H, Yoshida. Re-use of left internal thoracic artery for redo operation after MIDCAB. J Cardiovasc Surg 2000;41:399-400

\title{
Open-window thoracostomy and microvascular muscle flap for severe intrathoracic infection around aortic prosthetic graft
}

\author{
Nai-Chen Cheng, MD, Jiun Hsu, MD, Jing-Shing Chen, MD, Hao-Chih Tai, MD, and Hsi-Yu Yu, MD, \\ Taiwan, Republic of China
}

A

74-year-old male patient was transferred to our institute with a history of persistent fever after 6 weeks' antibiotic treatment for mycotic aortic aneurysm. Imaging study revealed multiple ruptures of the descending aorta, with pseudoaneurysm formation and marked inflammation (Figure 1, A). Blood culture yielded group D Salmonella species. Surgical debridement and replacement of the descending aorta from the high thoracic level to the level of the 10th intercostal artery with a knitted double-velour graft (Hemashield; Boston Scientific Corp, Natick, Mass) was performed under cardiopulmonary bypass and temporary hypothermic circulatory arrest through a lateral thoracotomy. Unfortunately, fever relapsed 10 days after the operation despite continuous antibiotic use. Three weeks postoperatively, follow-up computed tomography revealed severe periprosthetic abscess accumulation (Figure 1, B). Because the medical treatment appeared ineffective, we designed a 2 -stage surgical procedure to control the infection.

Meticulous debridement of necrotic and infected tissue was performed through the previous thoracotomy. After partial resection of 2 ribs, an open-window thoracostomy was created by using the Eloesser flap technique (Figure 2). The aortic pros-

From the Department of Surgery, National Taiwan University Hospital and National Taiwan University College of Medicine, Taipei, Taiwan, Republic of China.

Received for publication Aug 31, 2004; revisions received Sept 9, 2004; accepted for publication Sept 22, 2004.

Address for reprints: Hsi-Yu Yu, MD, Department of Surgery, National Taiwan University Hospital, 7 Chung-Shan South Rd, Taipei, Taiwan, Republic of China (E-mail: hsiyuyu@ @a.mc.ntu.edu.tw).

J Thorac Cardiovasc Surg 2005;129:1182-4

$0022-5223 / \$ 30.00$

Copyright $(\odot 2005$ by The American Association for Thoracic Surgery doi:10.1016/j.jtcvs.2004.09.024 thetic graft was exposed in the thoracostomy cavity, which was later filled with iodine-soaked pads. The dressing was changed every 8 hours at bedside, and the patient's fever gradually subsided. Two weeks later, when the local condition of the thoracostomy cavity appeared much improved, further infection control and obliteration of the cavity were achieved with a $25 \times$ $12 \times 6-\mathrm{cm}$ free vastus lateralis muscle flap harvested from his left thigh. The flap survived well, and the patient was discharged in stable condition 3 months after the initial operation (Figure 1, $C$, and 2,B). At 6 months of outpatient follow-up, the patient was well and without recurrence of intrathoracic infection with long-term oral antibiotics.

\section{Discussion}

Open-window thoracostomy is a well-established procedure for chronic empyema and other refractory intrathoracic infections. The subsequent large thoracic cavity might require microvascular muscle flap transfer. ${ }^{1}$ Whether this 2-stage surgical procedure can be used safely and effectively in patients with periprosthetic infection after surgical treatment of mycotic aortic aneurysm has not been described before. The presented case demonstrated its successful application in the management of severe intrathoracic infection around the aortic prosthetic graft.

Mycotic aortic aneurysm was reported to have a high operative and follow-up mortality rate, especially in patients presenting with ruptures of the aorta. ${ }^{2}$ Although recurrent infections are not uncommon, immediate surgical debridement and in situ grafting was considered the only treatment option for this patient because of his uncontrollable infection $^{3}$ and the proximity of the ruptures to the aortic arch. $^{4}$

For management of aortic prosthetic graft infection, several methods were reported in the literature, including in situ homograft replacement and extra-anatomic grafting. ${ }^{2}$ Because the aortic graft is in the vicinity of the aortic arch in this case, replacement of the infected graft necessitates another major operation with cardiopulmonary bypass and circulatory arrest, which could be devastating considering the patient's unfavorable clinical condition. Therefore, we applied the 

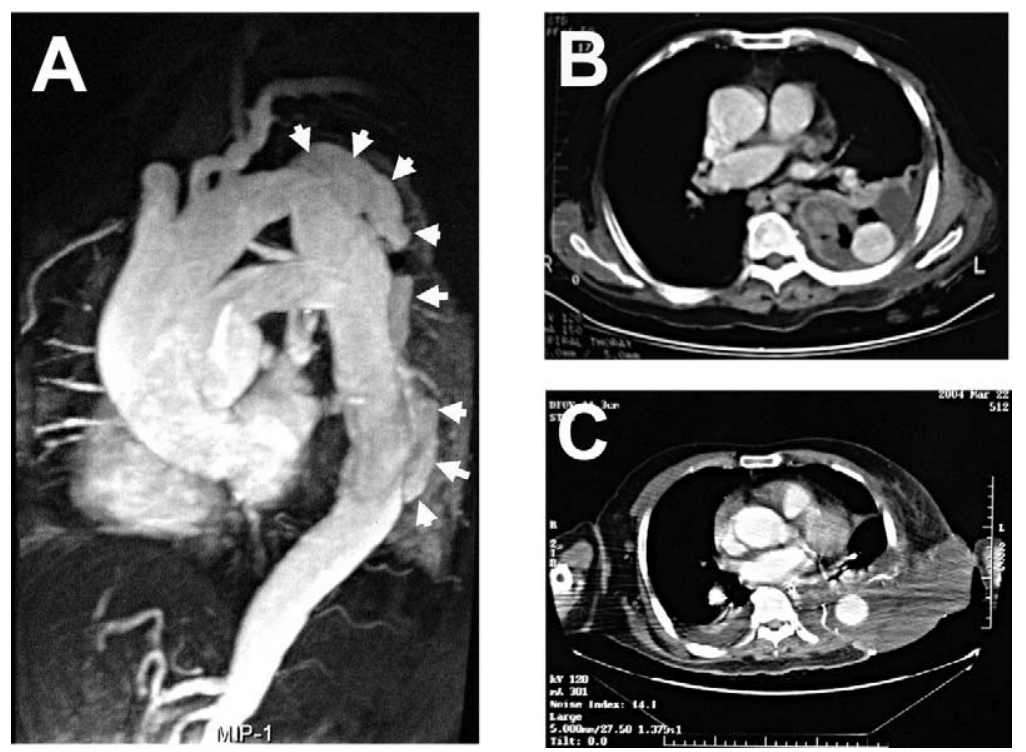

Figure 1. A, Magnetic resonance imaging-reconstructed angiogram before the initial operation revealed multiple ruptures of the thoracic aorta (indicated by arrowheads). B, Computed tomography performed 3 weeks after the initial surgical operation revealed severe infection around the prosthetic graft of the thoracic aorta. C, Computed tomography performed before discharge from the hospital revealed good myocutaneous flap growth and no residual abscess.

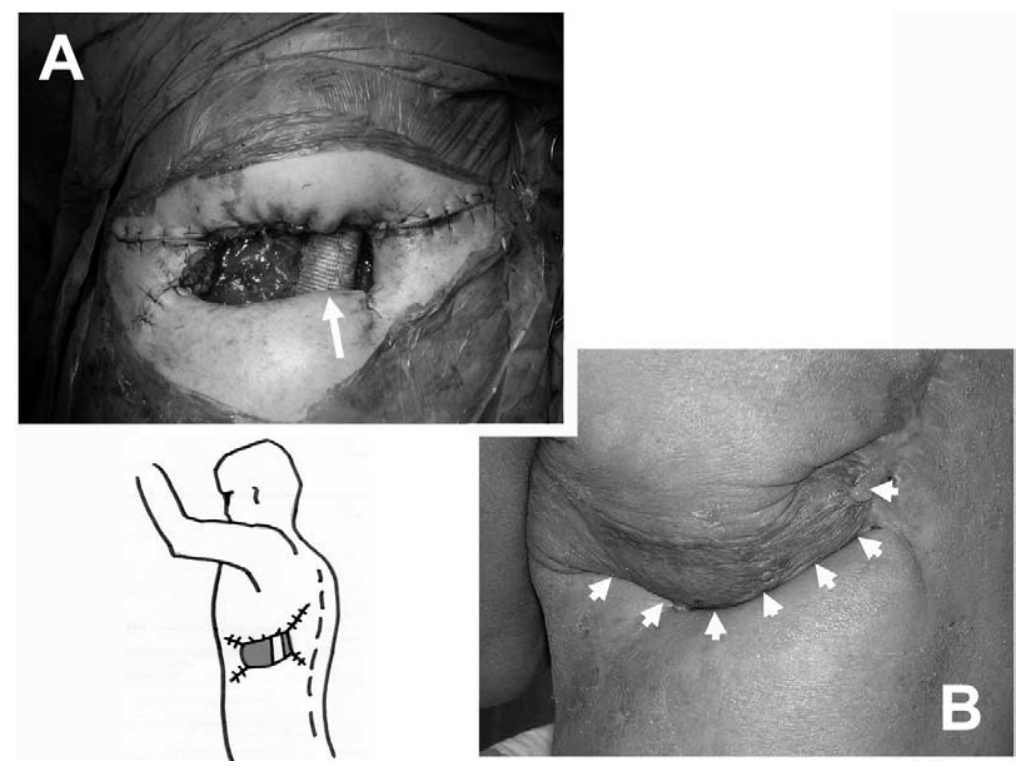

Figure 2. A, An open-window thoracostomy created by using the Eloesser flap technique at a previous left lateral thoracotomy wound. A prosthetic aortic graft can be seen in the thoracostomy cavity (indicated by arrow). B, The thoracostomy cavity was obliterated with a free vastus lateralis muscle flap (indicated by arrowheads).

old method (open-window thoracostomy and free muscle flap) to a new indication in a hope of getting the infection under control.

Muscle flap transposition is a valid therapeutic option in the management of intrathoracic infection with cavity formation. It not only obliterates the cavity but also delivers a high level of oxygen, antibiotics, and immunocompetent cells to the infected area. When major local muscles, such as pectoralis major and lattismus dorsi, have been divided in the course of previous thoracotomy or when they 
exhibit insufficient volume, a microvascular muscle flap is probably the best treatment option for reconstruction of a large thoracic dead space. The vastus lateralis muscle represents an ideal choice because its distal location allows simultaneous flap harvest and recipient site preparation. A large bulk can be provided to totally obliterate the space around the prosthetic graft, and there is minimal dysfunction of the lower limb after removal of the muscle. ${ }^{1}$

In conclusion, a 2-stage procedure with an open-window thoracostomy, followed by a microvascular muscle flap, might be of value to treat severe intrathoracic infection around aortic prosthetic grafts in high risk patients.

\section{References}

1. Chen HC, Santamaria E, Chen HH, Cheng MH, Chang CJ, Tang YB. Microvascular vastus lateralis muscle flap for chronic empyema associated with a large cavity. Ann Thorac Surg. 1999;67:866-9.

2. Muller BT, Wegener OR, Grabitz K, Pillny M, Thomas L, Sandmann $\mathrm{W}$. Mycotic aneurysms of the thoracic and abdominal aorta and iliac arteries: experience with anatomic and extra-anatomic repair in 33 cases. J Vasc Surg. 2001;33:106-13.

3. Hsu RB, Tsay YG, Wang SS, Chu SH. Management of aortic aneurysm infected with Salmonella. Br J Surg. 2003;90:1080-4.

4. Nakamura Y, Kawachi K, Imagawa H, Watanabe Y, Hamada Y, Tsunooka N. Mycotic aneurysm of the aortic arch due to Salmonella. Jpn J Thorac Cardiovasc Surg. 2003;51:253-5.

\section{Novel knot-tying technique for mitral valve repair}

Hironori Izutani, MD, PhD, Masao Yoshitatsu, MD, PhD, Jyun Kawamoto, MD, and Keijiro Katayama, MD, Hiroshima, Japan

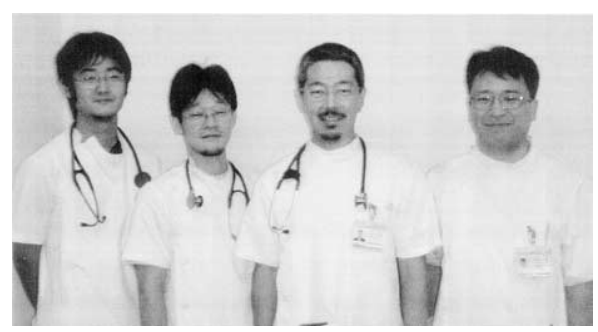

Katayama, Kawamoto, Izutani, Yoshitatsu (left to right)
$\mathrm{M}$ itral valve repair is a widely accepted procedure that now allows reconstruction in most patients with mitral regurgitation. ${ }^{1}$

Techniques in repair, such as quadrangular resection, sliding plasty, annular plication, and annuloplasty, are well performed. ${ }^{2}$ Chordal reconstruction through placement of artificial chordae is also one of the techniques for repair of the prolapsed mitral leaflet to correct elongated chordae or replace ruptured chordae, causing mitral regurgitation. ${ }^{3}$ In placing artificial chordae, adjustment of chordal height is a key for successful mitral valve repair. ${ }^{4}$ Even the surgeon recognizes the optimal knotting position: overaggressive sliding of knots might happen when tying the suture because manual hand tying causes poor visualization of the knots as a result of the small and deep working place involved in the mitral valve procedure. We used a classical knot-tying device to avoid critical knot sliding and to obtain better

From the Division of Cardiovascular Surgery, National Hospital Organization Kure Medical Center, Hiroshima, Japan.

Received for publication Sept 2, 2004; accepted for publication Sept 22, 2004.

Address for reprints: Hironori Izutani, MD, PhD, Division of Cardiovascular Surgery, National Hospital Organization Kure Medical Center, 3-1 Aoyama-cho, Kure, Hiroshima 737-0023, Japan (E-mail: izutani@kurenh.go.jp).

J Thorac Cardiovasc Surg 2005;129:1184-6

$0022-5223 / \$ 30.00$

Copyright $\odot 2005$ by The American Association for Thoracic Surgery

doi:10.1016/j.jtcvs.2004.09.026 visualization for the surgeon and the assistants in performing chordal reconstruction procedures.

\section{Technique}

The device was developed by Dr Hideo Sakihara in 1935 at the former National Kure Hospital in Japan and was manufactured by Senko Medical Instrument Manufacturing Company (Tokyo, Japan). ${ }^{5}$ This device was initially designed for thoracic surgery, especially for operations of the hilar pulmonary vessels because of a deep and narrow surgical field. It has been used mostly in the fields of general surgery, gynecology, and thoracic surgery in Japan. This device is made of metal, and it can be simply autoclaved for regular reuse.

The surgeon holds the device in one hand, makes a knot in the suture, pushes down the knot, and then pulls both ends of the suture with the other hand (Figure 1). The surgeon simply repeats the same maneuver and makes sure the knots are tightened. The surgeon and his or her assistants are able to see the tying process and make sure of the correct position of the knots at all times, even in a deep and narrow surgical field. Therefore, the use of this device makes the surgeon comfortable in deep knot tying.

In our standard procedure for mitral valve repair requiring median sternotomy, left atriotomy was carried out after establishment of cardiopulmonary bypass and cardioplegic arrest. After exposing the mitral valve, chordal reconstruction was performed in the present case with anterior mitral valve leaflet prolapse (Figure 2, A). A pledget-supported double-needled CV-5 polytetrafluoroethylene suture (W. L. Gore \& Associates, Inc, Flagstaff, Ariz) was placed on the anterolateral papillary muscle, and the suture was tied with the device. Then both ends of the suture were placed on the anterior leaflet (Figure 2, B) and tied with the same device 